\title{
FREQUÊNCIA DE NEOPLASIAS CUTÂNEAS EM EQUINOS: ESTUDO RETROSPECTIVO DO LABORATÓRIO DE PATOLOGIA VETERINÁRIA DA UNIVERSIDADE FEDERAL DO PARANÁ
}

\author{
Lew Kan Sprenger ${ }^{1}$, Lorran Baeumle Gabardo', Larissa Wunsche Risolia ${ }^{1}$, Marcelo \\ Beltrão Molento ${ }^{1}$, Antônio Waldir Cunha da Silva ${ }^{1}$, Renato Silva de Sousa ${ }^{1}$ \\ 1 UFPR \\ Correspondência: Lew Kan Sprenger: lew.sprenger@gmail.com
}

\begin{abstract}
RESUMO: O objetivo deste estudo foi determinar a prevalência dos tumores cutâneos de equinos examinados pelo Laboratório de Patologia Veterinária (LABPV) da Universidade Federal do Paraná (UFPR), Campus Curitiba. Foram pesquisados os protocolos de biópsia de pele destes animais, no período de 1984 a 2008. Os dados utilizados foram diagnóstico morfológico, sexo, idade e raça. Das 201 amostras recebidas, $166(82,6 \%)$ eram de tumores cutâneos, sendo 150 (74,6\%) neoplásicos. A raça mais prevalente foi a Puro Sangue Inglês 112 animais (67,5\%); seguida da Crioula 29 (17,5\%); Quarto de Milha $15(9,0 \%)$ e Mangalarga Marchador 7 (4,2\%). Animais sem raça definida somaram 3 $(1,8 \%)$ do total. A faixa etária mais prevalente, em ordem decrescente, foi: a 1-5 anos com 108 equinos (65,1\%); $6-14$ anos 41 (24,7\%); cavalos com 15 anos ou mais $13(7,8 \%)$ e com menos um ano apenas 1 cavalo (0,6\%). Com relação ao sexo, $102(61,5 \%)$ eram fêmeas, $61(36,7 \%)$ eram machos e em casos o gênero $(1,8 \%)$ não foi informado. As neoplasia cutâneas mais prevalentes foram: sarcóide com 111 casos (66,87\%); carcinoma de células escamosas 12 (7,23\%); papiloma 11 $(6,63 \%)$ e outros tumores somaram $32(19,87 \%)$. A raça Puro Sangue Inglês apresentou a maior prevalência para sarcóide, carcinoma de células escamosas e papiloma; sendo 77/111 (69,4\%); 7/12 $(58,4 \%)$ e $8 / 11(72,7 \%)$, respectivamente.
\end{abstract}

Palavras-chave: dermatologia; histopatologia; oncologia

\section{FREQUENCY OF SKIN NEOPLASMS IN HORSES: RETROSPECTIVE STUDY FROM VETERINARY PATHOLOGY LABORATORY OF FEDERAL UNIVERSITY OF PARANA}

\begin{abstract}
The aim of this study was to determine the prevalence of skin tumors in horses examined in Laboratory of Veterinary Pathology (LABPV), Federal University of Paraná (UFPR), Campus Curitiba. Was searched the skin biopsy protocols of these animals, from 1984 to 2008. The data used were morphologic diagnosis, gender, age and race. From 201 samples, $166(82,6 \%)$ were skin tumors and $150(74,6 \%)$ being neoplastic. The race most prevalent was the Thoroughbred 112 animals $(67,5 \%)$; followed by Creole $29(17,5 \%)$; Quarter Horse $15(9,0 \%)$ and Mangalarga $7(4,2 \%)$. Animals breed totaled $3(1,8 \%)$. The most prevalent age group, in descending order, were 1-5 years with 108 horses $(65,1 \%) ; 6-14$ years $41(24,7 \%) ; 15$ years or more $13(7,8 \%)$ and horses with at least a year or so $1(0,6 \%)$. With regard to gender, $102(61,5 \%)$ were female; $61(36,7 \%)$ were males and 3 $(1,8 \%)$ was not described. The skin neoplasms were more prevalent: 111 cases of sarcoid $(66,87 \%)$; 12 squamous cell carcinoma $(7,23 \%) ; 11$ papilloma $(6,63)$ and other tumors amounted to 32 $(19,87 \%)$. The Thoroughbred had the highest prevalence of sarcoid, squamous cell carcinoma and papilloma, being $77 / 111(69,4 \%) ; 7 / 12(58,4 \%)$ and $8 / 11(72,7 \%)$ respectively..
\end{abstract}

Key Words: dermatology; histopathology, oncology 
Frequência de neoplasias cutâneas em equinos: estudo retrospectivo do laboratório de patologia veterinária da universidade Federal do Paraná

\section{INTRODUÇÃO}

$\mathrm{O}$ interesse em diagnosticar e solucionar os problemas dermatológicos em equinos está crescendo a cada ano. Isto se deve ao fato de que as lesões de pele nestes animais acarretam em perdas econômicas, além de serem também um problema estético (TAYLOR e HALDORSON, 2013). Estas lesões são uma das queixas mais frequentes dos médicos veterinários que trabalham com esta espécie (PILSWORTH e KNOTTENBELT, 2007). Em estudo realizado no Estados Unidos, foi demonstrado que $4,1 \%$ dos cavalos examinados tinham algum problema dermatológico (SCOTT, 2007).

A gravidade do problema está no fato que a maioria das referidas lesões encontradas são neoplásicas (MUELLER, 2005). A pele é o órgão dos equinos onde mais ocorrem casos de neoplasias, chegando a aproximadamente $50 \%$ dos casos (BRUM et al., 2010). Vários são os tipos de neoplasias comumente encontradas, entre as quais merecem destaque, devida a maior incidência, o sarcóide, fibrossarcoma, melanoma, carcinomas e granulomas (BARRELET, 2010). A severidade de cada um desses tumores depende de fatores como estado geral do animal, imunidade, localização da neoplasia, entre outros (MEUTEN, 2008).

São importantes os estudos epidemiológicos para conhecer as diferenças de prevalência quanto ao sexo, idade e raça dos equinos afetados (WOBESER et al., 2010). Com este conhecimento pode-se estabelecer linhas de pesquisa visando minimizar ou prevenir esta enfermidade em determinado grupo (BOGAERT et al., 2005). Poucos são os estudos que já foram realizados tanto no Brasil (SOUZA et al., 2011) como em outros países (FOY et al., 2002; Lloyd et al.,
2003; MUELLER, 2005), sendo que muitos destes são antigos (RAGLAND et al. 1970; MILLER e CAMPBELL, 1982).

O objetivo do presente estudo foi pesquisar a prevalência de tumores cutâneos em equinos diagnosticados pelo Laboratório de Patologia Veterinária (LABPV), pertencente ao Hospital Veterinário da Universidade Federal do Paraná (HV-UFPR), Campus Curitiba.

\section{MATERIAL E MÉTODOS}

Para a produção do banco de dados utilizados no presente trabalho, foram analisados os dados referentes aos protocolos de biópsia de pele de equinos, realizados pelo LABPV-UFPR, entre janeiro de 1984 e dezembro de 2008. Foram utilizados os protocolos que mostravam tumores cutâneos, sendo neoplásicos ou não. Utilizaram-se os dados referentes ao diagnóstico, ao sexo, a idade e a raça do animal afetado. Fizeram parte do estudo apenas as lesões de pele, sendo que os aspectos histológicos das lesões não foram avaliados. Quanto ao sexo, os animais foram divididos apenas em machos e fêmeas, independentemente de serem castrados. Já para a qualificação quanto à idade, seguiramse os critérios etários estabelecidos por Egenvall et al. (2005), no qual foram estabelecidos quatro grupos: menores de 1 ano; 1 -5 anos; 6-14 anos e 15 anos ou mais. Por fim, foi calculada a prevalência dos tumores cutâneos utilizando o programa Epi Info ${ }^{\circledR}$ versão 3.5.1.

\section{RESULTADOS E DISCUSSÃO}

O período analisado foi de 1984 a 2008, sendo recebidas 201 amostras de biopsias de pele de equinos. Destas, $166(82,6 \%)$ eram de tumores cutâneos, 
sendo desses 150 (74,6\%) neoplásicos. Analisando a disposição quanto ao sexo, 102 (61,5\%) ocorreram em fêmeas, $61(36,7 \%)$ em machos e em 3 $(1,8 \%)$ não foi informado. Não existe uma correlação comprovada cientificamente quanto à predileção por sexo (FOY et al., 2002), sendo que este fato pode ser explicado pelo maior numero de animais do sexo feminino analisados.

O sarcóide foi a neoplasia mais prevalente neste estudo, 111 casos $(66,87 \%)$ (Quadro 1). Este resultado foi semelhante ao encontrado por Valentine (2006) no Estados Unidos, onde foi encontrado 51,4\%. Théon et al. (2007), examinando 630 tumores cutâneos encontrou $64,9 \%$ de prevalência para 0 sarcóide. Isso era esperado, pois esta é a neoplasia em equinos mais prevalente em muitos países (WOBESER et al., 2010). Não existe correlação entre sexo ou raça para a ocorrência deste tipo de neoplasia, contudo raças de sela têm maior predisposição a ter o sarcóide (SCOTT e MILLER, 2003). Animais que possuem maior contato com bovinos e consequentemente maior exposição ao papilomavírus também possuem maior predisposição a esta neoplasia (HAINISCH et al., 2012).

\begin{tabular}{|c|c|c|c|}
\hline Lesão tumoral & Quantidade & $\%$ & \%Acumulada \\
\hline Sarcóide & 111 & 66,87 & 66,87 \\
\hline $\begin{array}{c}\text { Carcinoma de células } \\
\text { escamosas }\end{array}$ & 12 & 7,23 & 74,10 \\
\hline Papiloma & 11 & 6,63 & 80,73 \\
\hline Pitiose & 9 & 5,42 & 86,15 \\
\hline Papiloma eosin ofílico & 6 & 3,62 & 89,77 \\
\hline Linfoma & 4 & 2,41 & 92,18 \\
\hline Fibrossarcoma & 3 & 1,81 & 93,99 \\
\hline Tecido de granulação & 3 & 1,81 & 95,80 \\
\hline Melanoma & 2 & 1,20 & 97,00 \\
\hline Granuloma eosinofílico & 2 & 1,20 & 98,20 \\
\hline Fibroma & 1 & 0,60 & 98,80 \\
\hline Calcinose circunscrita & 1 & 0,60 & 99,40 \\
\hline Nevo de colagéno & 1 & 0,60 & 100,00 \\
\hline
\end{tabular}

Neste estudo não foi encontrado nenhum caso de sarcóide em animais com menos de um ano de vida e nem com mais de 15 anos de idade (Quadro 2). Isto se deve ao fato que esta doença é mais prevalente em animais na faixa etária de três a seis anos (IRELAND et al., 2012). A maioria, 78,4\%\% (87/111), foi encontrada na categoria de 1-5 anos de idade. Os outros 19,8\% (22/111) foram incluídos no grupo com animais de 6-14 anos. Os resultados são semelhantes aos encontrados em outros estudos realizados nos Estados Unidos por Ginn et al. (2007) e no Brasil por Souza et al. (2011).

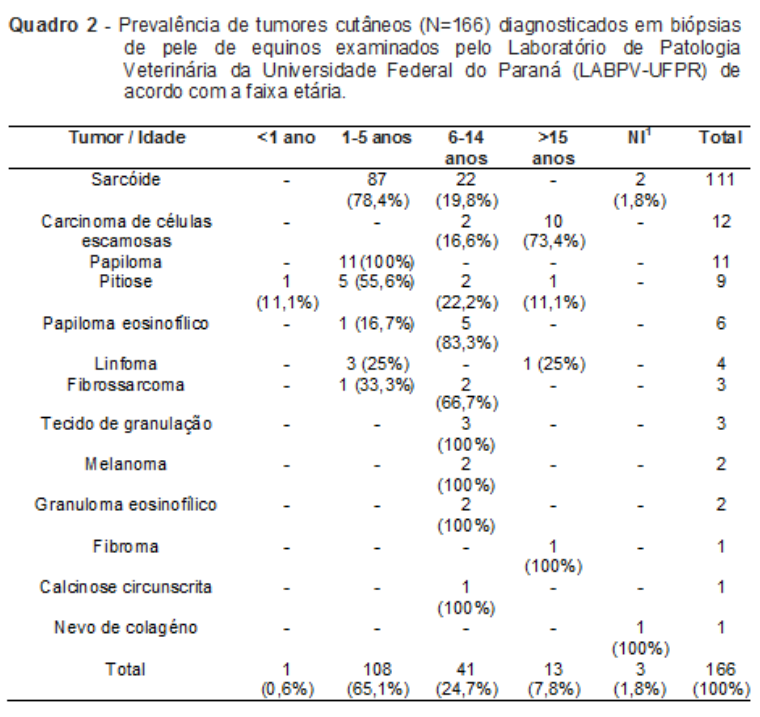

"Não Informado.

O segundo tumor com maior prevalência na pesquisa foi o carcinoma de células escamosas, representando $7,23 \%(12 / 166)$ do total. Esse resultado é semelhante ao encontrado por Valentine (2006), que observou 18,3\% de prevalência. Essa neoplasia é a segunda mais comum na maioria das localidades do mundo (FOY 2002; TAYLOR e HALDORSON, 2012), sendo a mais comum na pálpebra e anexos oculares (HENDRIX 2005) e também em prepúcio (VAN DEN TOP et al., 2008). Contudo a porcentagem de animais com este tipo de tumor pode aumentar, dependendo de fatores epidemiológicos do local, como idade e incidência de raios solares sobre os animais (BOLIN, 1999). Este tipo de carcinoma afeta animais mais velhos, normalmente com idade entre 12-18 anos (VAN DEN TOP et al., 2008). Em 
estudo realizado por Mair et al. (2000), a média de idade dos animais afetados por esse carcinoma foi de 17,4. Já Van der Zaag e Sloet Van OldruitenborghOosterbaan (2012) encontraram como média 16 anos. Na presente pesquisa a maior parte dos animais afetados $73,4 \%$ (10/12) estava no grupo de equinos com mais de 15 anos, estando a outra parcela $16,6 \%(2 / 12)$ no grupo de $6-14$ anos (Quadro 2).

Em terceiro lugar ficou o papiloma, apresentando 6,63\% (11/166). Este dado é conflitante com a literatura encontrada, onde Valentine (2006) encontrou nos Estados Unidos o melanoma (5,4\%) nesta posição, estando o papiloma em quarto lugar $(4,3 \%)$. Contudo no Brasil, Souza et al. (2011) observaram o papiloma como a terceira neoplasia mais relatada $(2,8 \%)$. Todavia em valores percentuais, esses três estudos apresentam valores semelhantes entre si. Para Smith et al. (2002), o papiloma é o terceiro tipo de tumor neoplásico mais importante em equinos, ficando atrás apenas do sarcóide e carcinoma de células escamosas. O papiloma ocorre normalmente em animais com cerca de três anos (BARRELET et al., 2010). Como descrito no Quadro 2, todos os animais com essa neoplasia cutânea (11/11) estão incluídas no grupo de 1-5 anos de idade. Souza et al. (2011) em seu estudo encontraram $2,8 \%$ de animais com papiloma e destes $100 \%$ estava inclusa na faixa etária de 1-5 anos.

A relação entre as raças e os tipos de tumores cutâneos está disposta no Quadro 3. Considerando o número total de neoplasias e também o número de casos isolados de sarcóide, carcinoma de células escamosas e papiloma, a raça Puro Sangue Inglês (PSI) apresentou a maior prevalência; sendo $69,4 \% \quad(77 / 111), \quad 58,4 \% \quad(7 / 12)$, $72,7 \%(8 / 11)$ e $67,5 \quad(112 / 166)$ respectivamente. Isso é explicado pelo alto número de animais desta raça que são atendidos no HV-UFPR. Seguindo a classificação geral, a segunda raça com maior prevalência foi a Crioula $17,5 \%$ (29/166); seguida da Quarto de Milha $9,0 \%(15 / 166)$ e Mangalarga 4,2\% (7/166). Animais sem raça definida somaram 1,8\% (3/166) do total. Apesar de alguns estudos apontarem uma correlação entre raça e predisposição a neoplasias cutâneas (ANGELOS et al., 1988), este fator ainda não foi bem esclarecido (FOY et al., 2002; EGENVALL et al., 2005). A proporção de animais afetados pode mudar dependendo das variáveis epidemiológicas existentes no ambiente onde os equinos estão inseridos (BARRELET et al., 2010).

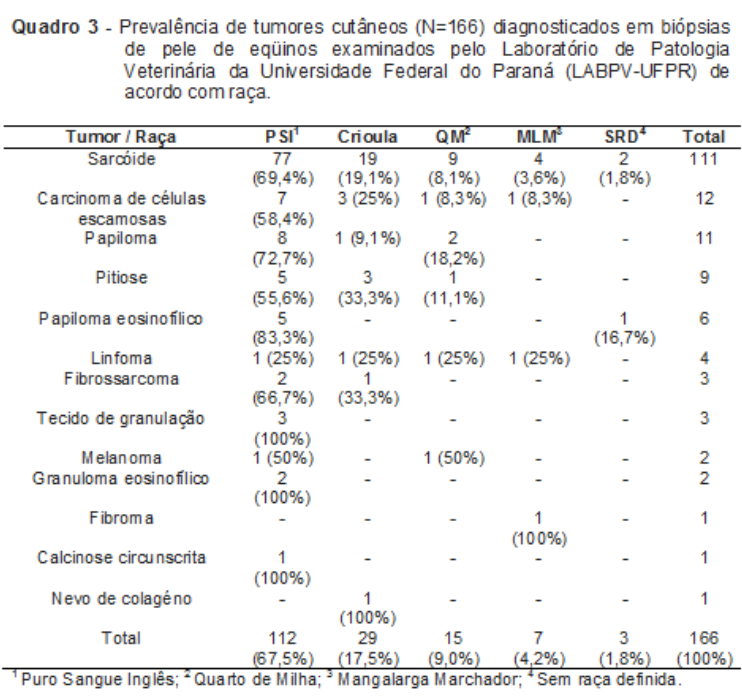

O tumor não neoplásico mais encontrado foi a pitiose $5,42 \%(9 / 166)$. Essa é uma doença granulomatosa causada pelo fungo Pythium insidiosum, tendo prevalência variada nas regiões tropicais e subtropicais do Brasil (SANTURIO et al., 2006). O segundo mais prevalente desta classe foi o tecido de granulação $1,81 \%(3 / 166)$; seguido de granuloma eosinofilico 1,2\% (2/166); calcinose circunscrita $06 \%(1 / 166)$ e nevo de colágeno $0,6 \%(1 / 166)$. Apesar de existirem alguns tumores de pele não 
neoplásicos que podem confundir o diagnóstico inicial das neoplasias cutâneas, com o exame histopatológico se pode diferenciá-las rapidamente (Meuten, 2008).

\section{CONCLUSÃO}

As principais neoplasias cutâneas de equinos diagnosticadas nas biopsias examinadas no LABPV-UFPR foram sarcóide, carcinoma de células escamosas e papiloma. A maior parcela dos animais afetados foi do sexo feminino, da raça Puro Sangue Inglês e na faixa etária de 6-14 anos. Os tumores não neoplásicos mais comuns foram pitiose, tecido de granulação exuberante e granuloma eosinofílico. Os dados encontrados no presente estudo servem como um importante informativo sobre os tumores cutâneos, uma vez que são escassos os trabalhos sobre este tema na literatura.

\section{REFERÊNCIAS}

BARRELET, A.; FOOTE, A; LITTLEWOOD, J.D. Common equine skin tumours. Companion Animal, v.15, p.9-17, 2010.

BOGAERT L. ; MARTENS A. ; DE BAERE C. et al. Detection of bovine papillomavirus DNA on the normal skin and in the habitual surroundings of horses with and without equine sarcoids. Research in Veterinary Science, v.79, p.253-258, 2005.

BOLIN, D.C. Cutaneous neoplasms of horses. Equine Disease Quarterly, v.7, n.4, p.7-9, 1999.

BRUM, J.S. ; SOUZA; T.M.; BARROS; C.S.L. Aspectos epidemiológicos e distribuição anatômica das diferentes formas clínicas do sarcoide equino no Rio Grande do Sul: 40 casos. Pesquisa Veterinária Brasileira, v.30, p.839-843, 2010.

EGENVALL, A.; PENELL, J.C.; BONNETT, B.N. et al. Morbidity of Swedish horses insured for veterinary care between 1997 and 2000: Variations with age, sex, breed and location. Veterinary Records, v.157, p.436-443, 2005.

FOY, J.M.; RASHMIR-RAVEN, A.M; BRASHIER, M.K. Common equine skin tumors. Compendium on Continuing Education for the Practising Veterinary, v.24, p.242-254. 2002.

GINN, P.E. ; MANSELL, J.E.K.L.; RAKICH P.M. Skin and appendages, In: Maxie, M.G. (Ed.), Jubb, Kennedy and Palmer's Pathology of Domestic Animals. 5th ed. Elsevier, Philadelphia, 2007, Cap x., p.553-781.

HAINISCH, E.K.; BRANDT, S.; SHAFTIKERAMAT, S. et al. Safety and immunogenicity of BPV-1 L1 virus-like particles in a dose-escalation vaccination trial in horses. Equine Veterinary Journal, v.44, p.107-111, 2012.

HENDRIX, D.V. Eye examination techniques in horses. Clinical Techniques in Equine Practice, v.4, n.1, p.2-10, 2005.

IRELAND, J.L.; WYLIE, C.E.; COLLINS, S.N. et al. Preventive health care and owner-reported disease prevalence of horses and ponies in Great Britain. Research in Veterinary Science, v.95, p.101-106, 2013.

LLOYD, D.H. Practical Equine Dermatology. 1st ed. Oxford: Blackwell Publishing Company Press, 2003. 137p.

MAIR, T.S.; WALMSLEY, J.P.; PHILLIPS, T.J. Surgical treatment of 45 horses affected by squamous cell carcinoma of the penis and prepuce. Equine Veterinary Science, v.32, n.5, p.406-410, 2000.

MEUTEN D. J. Tumors in Domestic Animals, $4^{\text {th }}$ ed. Raleigh: lowa State Press, 792 p. 2008.

MILLER, S.; CAMPBELL, R.S.F. A survey of granulomatous and neoplastic diseases of equine skin in north Queensland. Australian Veterinary Journal, v.59, n.2, p.33-37, 1982.

MUELLER, R.S. Dermatology for the Equine Practitioner. lowa: Ames, 2005. 97p.

PILSWORTH, R.C.; KNOTTENBELT , D. Skin Disease Refresher, Equine sarcoids. Equine Veterinary Education, v.5, p.260-262, 2007.

RAGLAND, W.L.; KEOWN, G.H.; SPENCER, G.R. Equine sarcoid. Equine Veterinary Journal. v.2, n.1, p.2-11, 1970.

SANTURIO, J.M.; ALVES, S.H.; PEREIRA, D.B. et al. Pitiose: uma micose emergente. Acta Scientiae Veterinariae, v.34, n.1, p.1-14, 2006.

SCOTT, D.W. Color Atlas of Farm Animal Dermatology. lowa: Blackwell, 2007. 252p.

SCOTT, D.W.; MILLER JR W.H. Equine

Dermatology. St. Louis: W.B. Saunders, 2003, p.698-795. 
SMITH, S.H.; GOLDSCHMIDT, M.H.;

MCMANUS, P.M. A comparative review of melanocytic neoplasms. Veterinary Pathology

Online, v.39, n.6, p.651-678, 2002.

SOUZA, T.M.; BRUM, J.S.; FIGHERA, R.A. et al. Prevalência dos tumores cutâneos de equinos diagnosticados no Laboratório de Patologia Veterinária da Universidade Federal de Santa Maria, Rio Grande do Sul. Pesquisa Veterinária Brasileira, v.31, n.5, p.379-382, 2011.

TAYLOR, S.; HALDORSON, G. A review of equine sarcoid. Equine Veterinary Education v.25, p.210-216, 2012.

TAYLOR, S.; HALDORSON, G. A review of equine mucocutaneous squamous cell carcinoma. Equine Veterinary Education, v. $\underline{25}$, p.374-378, 2013.

THÉON, A.P.; WILSON, W.D.; MAGDESIAN, K.G. et al. Long-term outcome associated with intratumoral chemotherapy with cisplatin for cutaneous tumors in equidae: 573 cases (19952004). Journal of American Veterinary Medicine Association, v.230, n.10, p.15061513, 2007.

WOBESER, B.K.; DAVIES, J.L.; HILL, J.E. et al. Epidemiology of equine sarcoids in horses in western Canada. Canadian Veterinary Journal, v.51, p.1103-1108, 2010.

VALENTINE, B.A. Survey of equine cutaneous neoplasia in the Pacific Northwest. Journal of Veterinary Diagnostic Investigation. v.18, p.123-126, 2006.

VAN DER ZAAG, E.J.; VAN

OLDRUITENBORGH-OOSTERBAAN, M.M.S.

Nodular skin problems encountered in a firstopinion equine clinic. Pferdeheilkunde, v.28, n.6, p.697-701, 2012.

VAN DEN TOP, J.; HEER, N.D.; KLEIN W.R. et al. Penile and preputial tumours in the horse: $\mathrm{A}$ retrospective study of 114 affected horses.

Equine Veterinary Education, v.40, p.528-532, 2008. 